\title{
PSICOLOGÍA
}

\section{Adolescentes escolarizados con intento de suicidio: oportunidad de intervención en colegios públicos y privados}

\author{
Carmen Marina Arrom Suhurt ${ }^{1}$, Mónica Ruoti ${ }^{1}$, \\ María Auxiliadora Arrom de Orrego", María del Pilar Fresco², \\ Margarita Samudio ${ }^{3}$, Cristina Arrom Suhurt ${ }^{3}$
}

\section{Resumen}

Introducción: A nivel mundial la depresión es una enfermedad frecuente y se calcula que afecta a más de 300 millones de personas. Puede convertirse en un serio problema de salud, principalmente si es de larga duración e intensidad moderada a grave, situación que puede causar mucho sufrimiento y afectar actividades laborales, escolares y familiares. Puede llevar al suicidio en el peor de los casos. Cada episodio de suicidio es una tragedia que aflige a familias, comunidades y países y tiene efectos perdurables para los allegados del suicida. Cada año se suicidan cerca de 800.000 personas y muchas más intentan hacerlo. El suicidio es considerado la tercera causa de defunción en el mundo. Constituye la segunda causa principal de defunción en el grupo etario de 15 a 29 años en la Región de las Américas en un $22 \%$. Un $75 \%$ de todos ellos ocurre en países de bajos y medianos ingresos. El estudio comparativo sobre mortalidad por suicidios en Colombia y México mostró que en el periodo analizado (20002013) la mortalidad por suicidios en los últimos años disminuyó de manera paulatina en Colombia, principalmente entre los 15 y 29 años en hombres, y en mujeres se distribuyó en diferentes grupos de edades, mientras en México el aumento incluyó todas las edades de manera constante En el primer semestre de 2016 se registraron en Paraguay 190 casos de suicidios, con un leve aumento con respecto al año anterior (187 casos), fluctuando la edad de las víctimas entre 18 a 29 años.

Objetivo: Se propuso describir características de una población adolescente escolarizada con antecedentes de intento de suicidio.

Material y Método: Se llevó a cabo un estudio transversal, descriptivo y

1. Centro para el Desarrollo de la Investigación Científica CEDIC, Universidad Nacional de Asunción, Paraguay.

2. Cátedra de Psiquiatría de la Facultad de Ciencias Médicas, Universidad Nacional de Asunción, Paraguay.

3. Instituto de Investigaciones en Ciencias de la Salud, Universidad Nacional de Asunción, Paraguay.

Trabajo presentado en la Revista Científica Estudios e Investigaciones de la Universidad iberoamericana y en el XXXII Congreso Argentino de Psiquiatría en abril 2017, Buenos Aires, Argentina; Categoría Poster.

E-mail: marinaarrom@yahoo.com.ar

DOI: 10.26885/rcei.foro.2017.121 
retrospectivo. Base de datos de adolescentes de 56 colegios públicos y privados de las ciudades de Minga Guazú, Hernandarias, Presidente Franco y Ciudad del Este del Departamento de Alto Paraná en Paraguay. Muestreo probabilístico. Se completó un cuestionario auto-administrado, estructurado y anónimo. Para conocer nivel de satisfacción con aspectos de su vida, se utilizó el Plan de Acción de Desarrollo y Salud de adolescentes y jóvenes en las Américas OPS/OMS. La información fue cargada en Excel, analizada con SPSS 15.0-IBM.

Resultados: Del total de la población de estudiantes (661), 16,6\% (110) manifestó que intentó quitarse la vida alguna vez. El 46,4\% (51) era de sexo masculino y el 53,6\%(59) femenino. El 20,2\% (22) de los entrevistados tenía de 12 a 14 años, el 61,7\% (68) de 15 a 17 años y un 17,2\% (19) 18 años y más. Se encontraba cursando la Educación Escolar Básica el 49,1\% (54) y el $50 \%$ (55) de los estudiantes el Bachillerato. Pertenecía a colegios públicos el $61,8 \%(68)$ y el $30,9 \%$ (34) a colegios privados. Vivía con los padres el $67,3 \%$ (74) estudiantes, con la madre el $15,5 \%(17)$, con parientes $8,2 \%(9)$, con el encargado no pariente $6,4 \%$ (7) y con el padre $1,8 \%$ (2). Un 39,1\% (43) tenía algún miembro de su familia con intento de suicidio y el 53,6\% (59) manifestó que no. Respondió en primera consulta que sufrió abuso sexual el 11,8\% (13) y que no lo padeció el 88,1\% (96). Al reiterar la pregunta el 38,2\% (42) afirmó que padeció abuso sexual mientras el 59,1\% (65) dijo que no. Consultó alguna vez por problemas emocionales el 19,1\% (21), el 67,3\% (74) no lo hizo. Se encontró una Correlación significativa entre haber sido víctima de abuso sexual e intento de suicidio (Valor de $\mathrm{p}=0,000$ ).

Conclusiones: en su mayoría los estudiantes eran de sexo femenino, y pertenecían a colegios públicos, vivían con ambos padres sin otra actividad. En porcentaje considerable fueron víctimas de abuso sexual y tenían un familiar con intento de suicidio. Se evidenciaron situaciones graves de trastornos de salud mental sin ser detectadas institucionalmente como oportunidad para intervenir y evitar muertes adolescentes. Debemos considerar que las instituciones educativas son una instancia reconocida para llevar adelante programas preventivos de suicidio, dirigido a adolescentes que presentan el intento de suicidio como un factor de riesgo temprano. Está demostrado que la implementación de programas de prevención reduce la depresión. Una de las estrategias comunitarias eficaces para prevenirla son los programas escolares que promueven un modelo de pensamiento positivo entre los niños, niñas y adolescentes.

Palabras clave: adolescentes escolarizados, intento de suicidio, prevención ámbito educativo.

\section{Referencias}

OMS. (2017). Depresión. Organización Mundial de la Salud 2017. Nota descriptiva $N^{\circ}$ 369. Recuperado de http://www.who.int/mediacentre/ factsheets/fs369/es/ 
Adolescentes escolarizados con intento de suicidio. Arrom et al.

OMS. (2016). Suicidio. Recuperado de http://www.who.int/mediacentre/ factsheets/fs398/es/

Preocupante cantidad de casos de suicidios (31 de julio 2016). ADN Paraguayo. Recuperado de http://www.adndigital.com.py/ preocupante-cantidad-de-casos-de-suicidios/

Silva, D., Valdivia, M., Vicente, B., Arévalo, E., Dapelo, R. \& Soto, C. (2017). Intento de suicidio y factores de riesgo en una muestra de adolescentes escolarizados de Chile. Revista de psicopatología y psicología clínica, 22(1), 33-42. doi:10.5944/rppc.vol.22.num.1.2017.16170 